\title{
Contingency and congruency switch in the congruency sequence effect: a reply to Blais, Stefanidi, and Brewer (2014)
}

\author{
James R. Schmidt* \\ Department of Experimental Clinical and Health Psychology, Ghent University, Ghent, Belgium \\ ${ }^{*}$ Correspondence: james.schmidt@ugent.be \\ Edited by: \\ Tobias Egner, Duke University, USA \\ Reviewed by: \\ Mike Wendt, Helmut Schmidt University, University of the Federal Armed Forces Hamburg, Germany \\ Wim Notebaert, Ghent University, Belgium
}

Keywords: congruency sequence effect, Gratton effect, contingency learning, switch costs, conflict adaptation, cognitive control, attention, feature integration

\section{A commentary on}

The Gratton effect remains after controlling for contingencies and stimulus repetitions

by Blais, C., Stefanidi, A., and Brewer, G. (2014). Front. Psychol. 5:1207. doi: 10.3389/fpsyg.2014.01207

\section{INTRODUCTION}

The congruency sequence effect (CSE) is the observation that the congruency effect is reduced following an incongruent trial (Gratton et al., 1992). Generally, the CSE is interpreted in terms of conflict adaptation, the idea that participants decrease attention to the distracter and/or increase attention to the target after experiencing conflict (e.g., Botvinick et al., 2001). An alternative learning and memory account proposes that the CSE is instead due to basic learning confounds (for a review, see Schmidt, 2013). For instance, systematic differences in the types of feature repetitions that are possible in each cell of the design might produce a CSE (Mayr et al., 2003; Hommel et al., 2004). Schmidt and De Houwer (2011) considered two additional learning and memory biases: sequential contingencies and congruency switch costs. However, Blais et al. (2014) present data which they suggest argue against a role of these two biases. This article illustrates some issues with this work and suggests that contingency and congruency switch biases may play a role after all.

\section{CONTINGENCY ANALYSIS UNDERPOWERED}

Schmidt and De Houwer (2011) introduced the idea that sequential contingency biases might influence the CSE. Often, each distracter is presented more frequently in the congruent color than in each incongruent color. Unfortunately, this introduces a contingency, whereby words are predictive of the congruent response. Contingency biases are larger following an accurately predictive trial than following an incorrectly predictive trial (Schmidt et al., 2007). Thus, contingency biases can contribute to the CSE. Indeed, Mordkoff (2012) showed that, with feature repetitions removed, the CSE is present in a contingency-biased Simon task, but is not observed in a contingency-unbiased version of the same task.

Blais et al. (2014) report a reanalysis of verbal Stroop data in which each participant performed several blocks of trials with varying contingencies (as manipulated with proportions of congruent trials) from 5 to $95 \%$ in increments of $5 \%$ (though only 10 to $80 \%$ could be analyzed). Overall, CSEs were not reliable for most contingency levels. Critically, the CSE did not significantly increase as a function of contingency in response times. With these data, the authors argued that contingencies are unlikely to play a role in the CSE.

However, statistical power of the sample of 15 participants is a concern. Indeed, the slope was notably positive, but with considerable error, $B=0.438 \pm 0.382$, $t_{(14)}=1.15, p>0.25$. The $B$ parameter is the amount of change in the CSE for a $1 \%$ contingency increment (i.e., $2.2 \mathrm{~ms}$ for each $5 \%$ increment, and $31 \mathrm{~ms}$ overall). Though not significant, this represents a medium effect size $(\beta=0.315)$. As Figure 1 illustrates, the study only had high (0.8) power to detect a large effect size ( $\beta=0.661)$. As a further concern, contingencies were manipulated between blocks. Contingency biases are known to transfer across blocks (Schmidt et al., 2010), causing contamination. Thus, contingency biases were probably underestimated. Curiously, the slope for the errors was significant and in the predicted direction, $B=0.038 \pm 0.017, \quad t_{(14)}=2.26$, $p=0.040$. Though seemingly confirming a role of contingencies, the authors reasoned that this slope is difficult to interpret given that none of the CSE estimates for the various contingency levels were significant. This argument does not seem particularly convincing, only feeding concerns about statistical power.

\section{CONGRUENCY SWITCH HYPOTHESIS, REVISED}

Schmidt and De Houwer (2011) further considered the possibility that there might be encoding costs associated with "switching" from a congruent to an incongruent trial, or vice versa, relative to repeating the same type of trial. Thus, following an incongruent trial, incongruent trials will incur a benefit and congruent trials a loss. The reverse is true following a congruent 


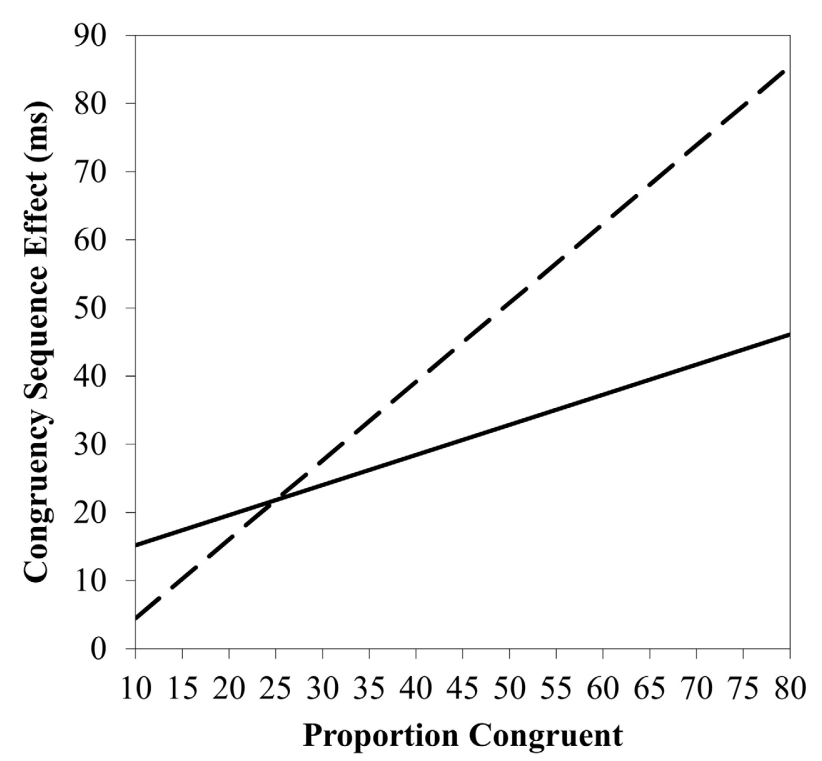

FIGURE 1 | Congruence sequence effect as a function of proportion congruency, with observed trend line (solid line) and trend line that would have been required for a high power test given the sample size and error (dashed line).

trial. As a result, congruency switch costs can further explain variance in the CSE.

Schmidt and De Houwer (2011) suggested that the cost of switching from congruent to incongruent might be "roughly" the same as the reverse, but Blais et al. (2014) did not observe this additivity. In retrospect, this was a misguided prediction. It is known that switching from a hard (non-dominant) to an easy (dominant) task sometimes incurs a larger cost on performance than the reverse, known as switch cost asymmetry (see Allport et al., 1994). The same might be predicted here, where a congruent "encoding shortcut" might be especially fast following a congruent trial, whereas the harder encoding task on incongruent trials will take long regardless of the previous trial congruency. This is an intriguing suggestion, because the conflict adaptation account should predict the exact opposite: because Stroop effects are primarily interference driven (see MacLeod, 1991), changes in attention to the word should be reflected primarily in incongruent trials.

Looking closely at the data of Blais et al. (2014), it can be seen that the interaction between congruency and congruency switch is due entirely to a larger effect of congruency switch for congruent trials (Experiment 1: $32 \mathrm{~ms}$; Experiment 2: $44 \mathrm{~ms}$ ) than for incongruent trials (Experiment 1: $-4 \mathrm{~ms}$; Experiment 2: $-15 \mathrm{~ms})$. Thus, this interaction is inconsistent with the conflict adaptation account, but is consistent with a revised version of the congruency switch hypothesis.

\section{CONCLUSION}

Though the current paper does not contest the notion that CSEs can be observed independent of feature repetition and contingency learning biases (e.g., Kim and Cho, 2014; Schmidt and Weissman, 2014; Weissman et al., 2014), three inferences of Blais et al. (2014) are contestable. First, contingency biases probably do play a role in the effect, as indicated by the significant effect in the errors, the underpowered but notable trend in the response times, and the data of Mordkoff (2012). Second, congruency switch effects might also play a role, as indicated by the direction of the switch cost asymmetry. Third, Blais and colleagues too quickly attribute the remaining CSEs to conflict adaptation. Yet other accounts still remain, such as the temporal learning and activation-suppression accounts, which actually seem to fit the extant data better than the conflict adaptation account (e.g., see Weissman et al., 2014, Schmidt and Weissman, in review).

\section{ACKNOWLEDGMENTS}

James R. Schmidt is a postdoctoral researcher of FWO - Vlaanderen (Research Foundation - Flanders).

\section{REFERENCES}

Allport, A., Styles, E. A., and Hsieh, S. (1994). "Shifting intentional set: exploring the dynamic control of tasks." in Attention and Performance XV: Conscious and Non-conscious Information Processing, eds C. Umiltà and M. Moscovitch (Cambridge, MA: MIT Press). 421-452.

Blais, C., Stefanidi, A., and Brewer, G. (2014). The Gratton effect remains after controlling for contingencies and stimulus repetitions. Front. Psychol. 5:1207. doi: 10.3389/fpsyg.2014.01207

Botvinick, M. M., Braver, T. S., Barch, D. M., Carter, C. S., and Cohen, J. D. (2001). Conflict monitoring and cognitive control. Psychol. Rev. 108, 624-652. doi: 10.1037/0033-295X.108.3.624

Gratton, G., Coles, M. G. H., and Donchin, E. (1992). Optimizing the use of information: strategic control of activation of responses. J. Exp. Psychol. Gen. 121, 480-506. doi: 10.1037/0096-3445 121.4.480

Hommel, B., Proctor, R. W., and Vu, K.-P. L. (2004). A feature-integration account of sequential effects in the Simon task. Psychol. Res. 68, 1-17. doi: 10.1007/s00426-003-0132-y

Kim, S., and Cho, Y. S. (2014). Congruency sequence effect without feature integration and contingency learning. Acta Psychol. 149, 60-68. doi: 10.1016/j.actpsy.2014.03.004

MacLeod, C. M. (1991). Half a century of research on the Stroop effect: an integrative review. Psychol. Bull. 109, 163-203. doi: 10.1037/00332909.109.2.163

Mayr, U., Awh, E., and Laurey, P. (2003). Conflict adaptation effects in the absence of executive control. Nat. Neurosci. 6, 450-452. doi: $10.1038 / \mathrm{nn} 1051$

Mordkoff, J. T. (2012). Observation: three reasons to avoid having half of the trials be congruent in a four-alternative forced-choice experiment on sequential modulation. Psychon. Bull. Rev. 19, 750-757. doi: 10.3758/s13423-012-0257-3

Schmidt, J. R. (2013). Questioning conflict adaptation: proportion congruent and Gratton effects reconsidered. Psychon. Bull. Rev. 20, 615-630. doi: 10.3758/s13423-012-0373-0

Schmidt, J. R., Crump, M. J. C., Cheesman, J., and Besner, D. (2007). Contingency learning without awareness: evidence for implicit control. Conscious. Cogn. 16, 421-435. doi: 10.1016/j.concog.2006.06.010

Schmidt, J. R., and De Houwer, J. (2011). Now you see it, now you don't: controlling for contingencies and stimulus repetitions eliminates the Gratton effect. Acta Psychol. 138, 176-186. doi: 10.1016/j.actpsy.2011.06.002

Schmidt, J. R., De Houwer, J., and Besner, D. (2010). Contingency learning in the blink of an eye: a resource dependent process. Conscious. Cogn. 19, 235-250. doi: 10.1016/j.concog.2009. 12.016

Schmidt, J. R., and Weissman, D. H. (2014). Congruency sequence effects without feature integration or contingency learning confounds. 
PLoS ONE 9:e102337. doi: 10.1371/journal.pone. 0102337

Weissman, D. H., Jiang, J., and Egner, T. (2014). Determinants of congruency sequence effects without learning and memory confounds. J. Exp. Psychol. Hum. Percept. Perform. 40, 2022-2037. doi: $10.1037 / \mathrm{a} 0037454$

Conflict of Interest Statement: The Associate Review Editor Wim Notebaert declares that, despite being affiliated to the same institution as the author James R. Schmidt, the review process was handled objectively and no conflict of interest exists. The author declares that the research was conducted in the absence of any commercial or financial relationships that could be construed as a potential conflict of interest.

Received: 07 October 2014; accepted: 17 November 2014; published online: 09 December 2014.

Citation: Schmidt JR (2014) Contingency and congruency switch in the congruency sequence effect: a reply to Blais, Stefanidi, and Brewer (2014). Front. Psychol. 5:1405. doi: 10.3389/fpsyg.2014.01405
This article was submitted to Cognition, a section of the journal Frontiers in Psychology.

Copyright (c) 2014 Schmidt. This is an open-access article distributed under the terms of the Creative Commons Attribution License (CC BY). The use, distribution or reproduction in other forums is permitted, provided the original author(s) or licensor are credited and that the original publication in this journal is cited, in accordance with accepted academic practice. No use, distribution or reproduction is permitted which does not comply with these terms. 